\title{
Anti-TNF $\alpha$ therapy in the management of psoriasis: experience of a state referral center ${ }^{*}$
}

\author{
Laura Maria Andrade Silva ${ }^{1}$ \\ Ana Cláudia Pinto Nobre ${ }^{1}$ \\ Ivonise Follador ${ }^{1}$
}

\author{
Bruno de Oliveira Rocha ${ }^{1,2}$ \\ Vitória Regina Pedreira de Almeida Rêgo ${ }^{1}$ \\ Maria de Fátima Santos Paim de Oliveira ${ }^{1}$
}

DOI: http://dx.doi.org/10.1590/abd1806-4841.20142613

\begin{abstract}
BACKGROUND: Psoriasis is a chronic immune-mediated disease, characterized by increased levels of $\mathrm{TNF} \alpha$. Anti-TNF $\alpha$ agents have revolutionized the treatment of severe psoriasis by targeting an important molecule involved in its pathogenesis.

OBJECTIVES: We report the experience of a state referral center that uses anti-TNF $\alpha$ agents for psoriasis.

MEtHODS: We conducted a retrospective case series. Seventy-four out of 120 patients met the inclusion criteria. Clinical and laboratory data was analyzed using the chi-squared, Wicoxon and McNemar's tests. Associations were considered statistically significant when $p$-value $<0.05$.

RESULTS: Forty-one subjects (55.40\%) were male, with a mean age of $47.69 \pm 14.99$ years. Median disease duration and pre-treatment PASI were 14.0 months (IQR 9.0-20.0), and 13.55 points (IQR 8.5-20.32). Sixty patients (81.10\%) had arthropathic psoriasis. Forty-six subjects $(62.20 \%)$ had comorbidities; the most frequent was dyslipidemia (25.70\%). In $55.40 \%$ of patients, insufficient response to conventional therapies was the principal indication for using anti-TNF $\alpha$ drugs. Clinical improvement occurred in $93.20 \%$ of cases, and the post-treatment PASI median was 0.0 points (IQR $0.0-0.0$ ). Adverse effects occurred in $6.80 \%$ of patients. Infections and elevation of transaminases occurred in $28.40 \%$ and $8.10 \%$ of cases, respectively.

CONCLUSION: Post-treatment reduction in PASI was satisfactory and the occurrence of adverse effects was minor, mostly mild infusion effects and local reactions at drug administration sites.
\end{abstract}

Keywords: Psoriatic arthritis; Psoriasis; Tumor necrosis factor-alpha

\section{INTRODUCTION}

Psoriasis is a chronic immune-mediated disease, characterized erythematous scaly lesions and is currently recognized as an inflammatory disorder with systemic impact, whose pathogenesis involves the deregulation of lymphocyte function, whereas the clinical repercussions of the disease are caused by signaling processes that culminate in abnormal proliferation of keratinocytes. ${ }^{1-4}$ These factors mean that psoriasis can be considered a prototype of Th1/Th17 disease: increased pro-inflammatory cytokines, such as IL2, INFy and TNFa, and decreased anti-inflammatory cytokines, such as IL10. ${ }^{5}$ Parallel to these findings, psoriasis treatment has progressed considerably, targeting the specific immunological events of this condition. Biopharmaceuticals represent an alternative to conventional treatment for severe and resistant forms of the disease. The Dermatology Service of the Complexo Hospitalar Universitário Prof. Edgar Santos (C-HUPES/UFBa) introduced the use of these drugs in the Brazilian Northeast in 2005, for the treatment of psoriatic arthritis and plaque psoriasis. The present study describes the experience of seven years of immunobiological drugs for psoriasis in our outpatient clinic.

Received on 12.03.2013.

Approved by the Advisory Board and accepted for publication on 29.07.2013

* Work performed at the Serviço de Dermatologia do Complexo Hospitalar Universitário Prof. Edgard Santos- Universidade Federal da Bahia (HUPES/UFBA) - Salvador (BA), Brasil.

Financial Support: None

Conflict of Interest: None

Universidade Federal da Bahia (UFBA) - Salvador (BA), Brazil.

University of Toronto - Toronto (ON), Canada.

(C)2014 by Anais Brasileiros de Dermatologia 


\section{METHODS}

A case series was conducted with patients attending the outpatient clinic of the Psoriasis C-HUPES/UFBa in Salvador, Bahia, Brazil, including patients with regular follow-up at the service and using immunobiological agents for at least 90 days. Clinical and laboratory data were collected through patients' medical records, which included age, gender, time of disease, clinical form of psoriasis, previous treatment, familial history, PASI, history of infections, side effects during treatment with biologic, comorbid conditions, serum transaminases and others. The study protocol was duly approved by the Research Ethics Committee of the establishment.

The data were stored and analyzed with SPSS $^{\circledR} 18.0$ for Windows. Descriptive statistics were used to characterize the population studied (mean, standard deviation, absolute and relative frequency). Median and interquartile ranges (IQR) were used for variables with asymmetrical distribution (time of disease, treatment duration and PASI). To investigate the association between the variables, we used the chisquared, Wicoxon and McNemar's tests, and associations were considered significant when the calculated $p$-value was lower than 0.05 .

\section{RESULTS}

The medical records of 120 patients treated in our outpatient clinic were assessed and 74 met the previously established inclusion criteria. Forty-one patients $(55.40 \%)$ were male and the mean age of the population studied was $47.69 \pm 14.99$ years, ranging from 13.0-92.0. The median time of disease was 14.0 months (IQR 9.0-20.0). The most prevalent clinical form was arthropathic psoriasis, in 60 patients $(81.10 \%)$. Only 8 subjects $(10.85 \%)$ had a positive familial history for the condition. The summarized clinical data for these patients is displayed in table 1.

Comorbidities were observed in 46 patients $(62.20 \%)$, and dyslipidemia was the most common (19 subjects, $25.70 \%$ ). The frequencies of the comorbid conditions found in our patients are shown in table 2.

Seventy-one patients $(95.90 \%)$ had been treated with traditional therapies before the indication of immunobiologics. Forty-nine individuals (66.20\%) were treated simultaneously with methotrexate, in doses ranging from 10 to $15 \mathrm{mg} /$ week. Other therapeutic modalities used with anti-TNFa agents included acitretin, cyclosporine and phototherapy. Table 3 illustrates the frequencies of their utilization with and before biologic therapy.

The main indication for therapy with immunobiologics was an insufficient response to standard therapies, in $55.40 \%$ (41) of patients. The combination of insufficient response or toxicity to conventional
TABle 1: Summarized clinical data of the 74 patients. Age is described as mean \pm standard deviation, and time of disease is displayed as median and interquartile range. All other variables are displayed as relative and absolute frequency (outside and inside the parentheses, respectively)

\begin{tabular}{|c|c|c|}
\hline \multicolumn{3}{|l|}{ Gender } \\
\hline & Male & $55.4 \%(41)$ \\
\hline & Female & $44.6 \%(33)$ \\
\hline \multicolumn{3}{|c|}{ Age (years) } \\
\hline & Mean & $47.69 \pm 14.99$ \\
\hline & Range & $13.0-92.0$ \\
\hline \multicolumn{3}{|c|}{ Time of disease (years) } \\
\hline & Median & 14 \\
\hline & Interquartile range & $9,0-20,0$ \\
\hline \multicolumn{3}{|c|}{ Comorbid conditions } \\
\hline & Yes & $62.2 \%(46)$ \\
\hline & No & $37.8 \%(28)$ \\
\hline \multicolumn{3}{|c|}{ Clinical forms of disease } \\
\hline & Arthropathic & $81.1 \%(60)$ \\
\hline & Vulgar & $16.2 \%(12)$ \\
\hline & Palmoplantar & $1.4 \%(1)$ \\
\hline & Pustular & $1.4 \%(1)$ \\
\hline \multicolumn{3}{|c|}{ Familial history for psoriasis } \\
\hline & Positive & $10.85 \%(8)$ \\
\hline & Negative & $89.12 \%(66)$ \\
\hline
\end{tabular}

TABLE 2: Frequency of comorbid conditions in the 74 patients. Relative and absolute frequency are displayed outside and inside the parentheses, respectively

\begin{tabular}{lll}
\hline Comorbid condition & \multicolumn{2}{l}{ Frequency } \\
& & \\
Dyslipidemia & $25.7 \%$ & $(19)$ \\
Arterial hypertension & $24.3 \%$ & $(18)$ \\
Depression & $13.5 \%$ & $(10)$ \\
Diabetes mellitus & $12.2 \%$ & $(9)$ \\
Obesity/overweight & $6.8 \%$ & $(5)$ \\
Atopy & $1.4 \%$ & $(1)$ \\
Others* & $24.3 \%$ & $(18)$ \\
No comorbid condition & $37.8 \%$ & $(28)$ \\
\hline
\end{tabular}

*Other comorbidities include hepatitis C, HTLV infection, nephrolithiasis, bipolar disorder, coronary artery disease, heart failure, portal fibrosis, renal failure, osteopenia and hydronephrosis.

therapies and disease severity accounted for 37.80\% (28) of the reasons for indication. Individually, the toxicity of other treatments and severity of psoriasis accounted for $4.10 \%$ (3) and $2.40 \%$ (2) of indications, respectively. 
TABLE 3: Frequency of use of other therapeutic modalities before and during treatment with anti-TNF $\alpha$ agents

\begin{tabular}{lcllll} 
& \multicolumn{2}{c}{ During anti-TNF $\boldsymbol{\alpha}$ treatment } & \multicolumn{2}{c}{ Before anti-TNF $\boldsymbol{\alpha}$ treatment } & p-value* \\
\hline Methotrexate & 42 & $(56.75 \%)$ & 65 & $(87.83 \%)$ & 0.00116 \\
Acitretin & 0 & $(0 \%)$ & 33 & $(44.59 \%)$ & 0.00011 \\
Cyclosporine & 0 & $(0 \%)$ & 33 & $(44.59 \%)$ & 0.00011 \\
PUVA & 1 & $(1.35 \%)$ & 19 & $(25.67 \%)$ & 0.00000 \\
\hline
\end{tabular}

*McNemar's Test

The immunobiologicals available in our service are infliximab, etanercept and adalimumab, with the following, respective, frequency rates of use: $48.60 \%$ (36 patients), $32.4 \%$ (24 patients) and $18.90 \%$ (14 patients). The median treatment period with antiTNFa agents was 18.0 months (IQR 9.0-36.0). These data are shown in table 4 . The median pre-treatment PASI was 13.55 points (IQR 8.5-20.32), and the posttreatment PASI was 0.0 points (IQR $0.0-0.0$ ), measured after completing treatment or at the last consultation (Table 5). Clinical improvement occurred in $93.20 \%$ (69) patients, taking into account the PASI (Wicoxon test $p$-value $=0,062$ ).

Of the 74 patients included, 14 (18.90\%) needed to change their immunobiologic agent, the main reason being insufficient responses to the drugs used, in $13.50 \%$ (10 patients). Three patients $(4.10 \%)$ had indication to change the immunobiologic due to side effects. One patient $(1.40 \%)$ had his medication substituted due to a combination of these two factors. A second change of immunobiologic drug was necessary in 3 patients $(4.10 \%)$, and a third in 1 patient $(1.40 \%)$, all motivated by an unsatisfactory response to therapy. Adverse events occurred in 5 patients $(6.80 \%)$, the most common being infusion reactions in 4 patients ( $80 \%$ of them)

During the assessment to indicate treatment with immunobiologics, 19 patients $(25.70 \%)$ tested positive for tuberculin, who then underwent 6 months of chemoprophylaxis with isoniazid. There were no cases of active infection with the tuberculosis bacillus.

TABLE 4: Distribution of anti-TNF $\alpha$ treatment according to frequency and duration of treatment (as mean \pm standard deviation) in months

\begin{tabular}{cl}
\hline & \multicolumn{1}{c}{ Frequency } \\
\hline Anti-TNF $\boldsymbol{\alpha}$ agent used & \\
Infliximab & $48.6 \%(36)$ \\
Etanercept & $32.4 \%(24)$ \\
Adalimumab & $18.9 \%(14)$ \\
& \\
Duration of treatment (months) & $22.06 \pm 15.7$ \\
Mean & $3-72$ \\
Range &
\end{tabular}

TABLE 5: PASI values before and after treatment with anti-TNF $\alpha$ agents, described as median and interquartile range

\begin{tabular}{lll}
\hline & PASI (points) & p-value* \\
\hline $\begin{array}{l}\text { Pre-treatment } \\
\text { Median }\end{array}$ & 13.55 & 0.062 \\
$\quad$ Interquartile range & $8.5-20.32$ & \\
& & \\
Post-treatment & 0.0 & \\
$\quad$ Median & & \\
$\quad$ Interquartile range & $0.0-0.0$ & \\
\hline
\end{tabular}

*Wicoxon test.

During treatment, infections occurred in $28.40 \%$ (21) of patients. The most common was respiratory tract infection $(12.20 \%$; 9 patients), followed by urinary tract infection $(2.70 \% ; 2$ patients), pneumonia $(2.70 \%$; 2 patients) and other infections (10.80\%; 10 patients).

Elevated hepatic enzymes in amounts at least twice greater than the reference value were observed in $6(8.10 \%)$ patients. The presence of comorbid conditions was positively correlated with the occurrence of transaminase elevations (chi-squared test $p$-value $<0.05$ ), and 3 were taking concomitant methotrexate.

\section{DISCUSSION}

The advent of immunobiological agents revolutionized the treatment of psoriasis. Our outpatient clinic is the state referral center in dermatology, performing the treatment of the vast majority of patients with moderate to severe psoriasis in Bahia, Brazil. Data from our series show that most patients using immunobiologics are middle-aged males, previously treated with more than one conventional therapy and having at least one comorbidity, particularly hypertension and dyslipidemia. Compared with the general population, patients with psoriasis are more likely to have associated comorbities, including heart disease, depression, arthritis, hypertension and dyslipidemia. ${ }^{6}$ A study carried out at our institution has demonstrated that psoriasis is associated with overweightness and obesity, the etiopathogenic link being chronic and mild systemic inflammation. ${ }^{7}$ 
Psoriatic arthritis is considered a common manifestation, observed in $40 \%$ of patients with moderate to severe psoriasis. In our study, the prevalence was much greater, affecting $81 \%$ of the subjects. The prevalence of depression in our patients was $13.5 \%$, considerably lower than the rate mentioned in publications from this field (about 32 to $60 \%$ ). ${ }^{8}$ We believe that this condition has been underestimated, requiring greater attention in order to intervene effetcively in improving the quality of life of patients.

Many indications for treatment were due to the safety profile of immunobiological agents regarding liver and kidney toxicity (31 patients; $41.90 \%$ ), contraindicating other therapies. The safety profile of anti-TNFa agents for patients with comorbidities was analyzed recently by Kimball et al. No significant difference has been demonstrated regarding changes in blood pressure, blood glucose or bodyweight, when compared with placebo. Also, the same study found no difference in the occurrence of serious adverse events among the same population. ${ }^{6}$

Biologics seem to be a safe choice for patients with chronic infections. Our series includes a 72-yearold man with psoriatic arthritis, and infected with the hepatitis $\mathrm{C}$ virus (HCV), who was successfully treated using etanercept for 3 years and infliximab for 2 years, without any complications registered thus far. The management of patients with hepatitis $C$ and psoriasis is seen as a major challenge, since most traditional therapies are hepatotoxic and/or immunosuppressive drugs, while the main treatment for HCV worsens psoriasis. ${ }^{9}$ Another patient of ours with positive serological tests for HTLV, has been on etanercept for 18 months without intercurrences.

One of our patients developed Hodgkin lymphoma during treatment with adalimumab, after 18 months using the medication. Once treatment was interrupted, the patient was taken to the hematologyoncology service, which initiated chemotherapy, with good responses. The risk of patients on adalimumabdeveloping this malignancy has been researched previously. Although the risk for patients with psoriasis vulgaris is equal to zero relative to the overall population, the respective values for rheumatoid arthritis, psoriatic arthritis, ankylosing spondylitis, Crohn's disease and juvenile idiopathic arthritis, are $0.12,0.2$, $0.08,0.08$ and $0.08 .^{10}$ Though further studies are required to clarify this association, we recommend caution with regard to this complication.

Adverse events were rare, occurring only in 5 $(6.75 \%)$ patients. In $60 \%$ (3) of these cases, gravity was enough to indicate achange of anti-TNFa. Though these events occurred predominantly during treatment with infliximab, there was no statistical significance in this association. Current literature suggests that most common adverse events are infusion reactions for infliximab, and injection site reactions for etanercept and adalimumab. ${ }^{11,12}$

A prospective study involving 174 patients carried out in Holland found that $28 \%$ of patients experienced serious adverse events related to biological therapy, including an increased risk of nonmelanoma skin cancer. ${ }^{13}$ In our experience with immunobiologics, no patient has developed skin cancer or presented serious adverse reactions.

Most patients remain on the same drug throughout treatment. At the time of analysis, 36 subjects were using infliximab, 24 were on etanercept, and 14 were using adalimumab. This distribution stems from several factors, including the first release and provision of infliximab and etanercept by the State Health Secretary (high-cost medications program). Meanwhile, loss of drug response was the main reason for patients being indicated for changes inimmunobiologics.

Despite belonging to the same pharmacological group, anti-TNFa agents have individual characteristics which differentiate them from one another, thus providing different results. The risk involved in patients receiving monoclonal anti-TNFa agents to produce neutralizing antibodies is well-known, and it could be a reason for loss of drug response, although we cannot confirm this in the subjects examined. The presence of these antibodies was detected in $49 \%$ of patients receiving adalimumab after 24 weeks of therapy. The degree of therapeutic response was also inversely proportional to the serum levels of these antibodies. ${ }^{14}$ The EXPRESS 2 study also correlated the presence of neutralizing antibodies to a decrease in clinical response to infliximab, though its mere presence is not regarded as constituting treatment failure. ${ }^{11,15,16}$ Regarding etanercept, it is known that up to $5 \%$ of patients develop antibodies against the medication. However, their involvement in drug efficacy is unclear. Thus, primary and secondary failures occur in response to all inhibitors of TNFa agents, but the presence of antibodies cannot be considered the only explanation for loss of effectiveness. ${ }^{17}$

Serum levels of the drug must also be considered in the context of a therapeutic failure. The infliximab dose is calculated per kilogram of bodyweight, while etanercept and adalimumab are administered infixed doses, which could explain an inadequacy of serum levels of the substance in certain patients. In a study by Van Lumiget al., of the 5 patients that used etanercept and adalimumab and experienced therapeutic failure, 3 were obese. They responded well to adalimumab with increased doses and frequency of injections. ${ }^{18}$ We believe that patients with significant obesity experience loss of response due to the inadequacy of the dose/body weight ratio. 
In our assessment, $93.2 \%$ of patients showed clinical improvement with the introduction of immunobiologic agents. This improvement was assessed by dermatological consultation, physical examination and improved quality of life. Although we did not observe a statistically significant difference between the pre- and post-treatment PASI (Wicoxon test $p$-value $=0.062$ ) in this study, this is probably due to the relatively small number of patients included and the inherent methodological limitations of a retrospective study.

Regarding the risk of infections, 21 patients presented infectious processes during treatment, and infection of the upper airways was the most prevalent. Although most infectious processes were mild, 1 patient treated with infliximab died due to urinary tract infection sepsis. It was an elderly individual with multiple comorbid conditions, whose medication was discontinued immediately after initiation of the clinical picture. Although there is a theoretical risk of an increase in infectious processes when treating with biological therapy, no studies comparing placebo with
TNFa inhibitors have shown a statistically significant increase in infection rates. ${ }^{11}$

In 6 patients, there was an increase in liver enzyme levels, all of which were under twice the reference value. In 3, methotrexate was associated with biological treatment, and they all had comorbid conditions (chi-squared $p$-value<0.05), 2 with schistosomotic portal fibrosis and 1 with a history of alcoholism.

\section{CONCLUSION}

Although immunobiologics are available for a considerable period of time, investigation into their long-term safety and side effects is still needed. The high costs associated with treatment and the fear of long-term effects remain important factors in selecting these drugs.

Finally, though the present study has several limitations inherent to a retrospective design, we emphasize the importance of disseminating local experiences of use of these drugs. $\square$

\title{
REFERENCES
}

1. Friedewald VE, Cather JC, Gelfand JM, Gordon KB, Gibbons GH, Grundy SM, et al. AJC editors' consensus: psoriasis and coronary artery disease. Am J Cardiol. 2008;102:1631-43.

2. Nickoloff BJ, Qin JZ, Nestle FO. Immunopathogenesis of psoriasis. Clin Rev Allergy Immunol. 2007;33:45-56.

3. Ghoreschi K, Weigert C, Röcken M. Immunopathogenesis and role of T cells in psoriasis. Clin Dermatol. 2007;25:574-80.

4. Kormeili T, Lowe NJ, Yamauchi PS. Psoriasis: immunopathogenesis and evolving immunomodulators and systemic therapies; U.S. experiences. $\mathrm{Br} J$ Dermatol. 2004;151:3-15

5. Krueger J. Determining the extent to which clinically effective treatment, ustekimumab or etanercept, reverses the molecular disease profile of psoriatic skin: comparison of lesional, non-lesional and a normal skin. AAD2011, New Orleans, P01.

6. Kimball AB, Bensimon AG, Guerin A, Yu AP, Wu EQ, Okun MM, et al. Efficacy and safety of adalimumab among patients with moderate to severe psoriasis with co-morbidities: Subanalysis of results from a randomized, double-blind, placebo-controlled, phase III trial. Am J Clin Dermatol. 2011;12:51-62.

7. Duarte GV. Associação entre obesidade, resposta imune e gravidade da Psoríase. [dissertação]. Salvador: (BA): Universidade Federal da Bahia; 2010. 966.

8. Leonard DG, O'Duffy JD, Rogers RS. Prospective analysis of psoriatic arthritis in patients hospitalized for psoriasis. Mayo Clin Proc. 1978;53:511-8.

9. Frankel AJ, Van Voorhees AS, Hsu S, Korman NJ, Lebwohl MG, Bebo BF Jr, et al. Treatment of psoriasis in patients with hepatitis C: from the Medical Board of the National Psoriasis Foundation. J Am Acad Dermatol. 2009;61:1044-55.

10. Burmester GR, Mease P, Dijkmans BA, Gordon K, Lovell D, Panaccione R, Adalimumab safety and mortality rates from global clinical trials of six immune-mediated inflammatory diseases. Ann Rheum Dis. 2009;68:1863-9.

11. Menter A, Feldman SR, Weinstein GD, Papp K, Evans R, Guzzo C. A randomized comparison of continuous vs. intermittent infliximab maintenance regimens over 1 year in the treatment of moderate-to-severe plaque psoriasis. J Am Acad Dermatol. 2007;56:31.e1-15.

12. Leonardi C, Papp K, Strober B, Reich K, Asahina A, Gu Y, et al. The long-term safety of adalimumab treatment in moderate to severe psoriasis: a comprehensive analysis of all adalimumab exposure in all clinical trials. Am J Clin Dermatol. 2011;12:321-37.

13. van Lümig PP, Driessen RJ, Berends MA, Boezeman JB, van de Kerkhof PC, de Jong EM. Safety of treatment with biologics for psoriasis in daily practice: 5-year data. J Eur Acad Dermatol Venereol. 2012;26:283-91.

14. Lecluse LL, Driessen RJ, Spuls PI, de Jong EM, Stapel SO, van Doorn MB, et al. Extent and clinical consequences of antibody formation against adalimumab in patients with plaque psoriasis. Arch Dermatol. 2010;146:127-32.

15. Vender R. An open-label, prospective cohort pilot study to evaluate the efficacy and safety of etanercept in the treatment of moderate to severe plaque psoriasis in patients who have not had an adequate response to adalimumab. J Drugs Dermatol. 2011;10:396-402.

16. Warren RB, Brown BC, Lavery D, Griffiths CE. Adalimumab for psoriasis: practical experience in a U.K. tertiary referral centre. Br J Dermatol. 2010;163:859-62.

17. Ormerod AD. Switching biologics for psoriasis. Br J Dermatol. 2010;163:667-9.

18. Van Lümig PP, Lecluse LL, Driessen RJ, Spuls PI, Boezeman JB, van de Kerkhof PC, et al. Switching from etanercept to adalimumab is effective and safe: results in 30 patients with psoriasis with primary failure, secondary failure or intolerance to etanercept. $\mathrm{Br} \mathrm{J}$ Dermatol. 2010;163:838-46.

\author{
MAILING ADDRESS: \\ Bruno de Oliveira Rocha \\ Rua Padre Feijó, 240 \\ Ambulatório Magalhães Neto, $3^{\circ}$. Andar \\ Canela \\ 40110-170 - Salvador - BA \\ Brazil \\ E-mail:brunorocha.17@hotmail.com
}

How to cite this article: Andrade Silva LM, Rocha BO, Nobre ACP, Rêgo VRPA, Follador I, Oliveira MFSP. Anti$\mathrm{TNF} \alpha$ therapy in the management of psoriasis: experience of a state referral center. An Bras Dermatol. 2014;89(3):436-40. 